\title{
Additional Imaging Following a Negative Sestamibi Scan in Primary Hyperparathyroidism
}

\author{
Bas Twigt ${ }^{1}$, Anne Vollebregt ${ }^{2}$, Piet de Hooge ${ }^{3}$, Alex Muller ${ }^{4}$, Thijs van Dalen ${ }^{1}$ \\ ${ }^{1}$ Department of Surgery, Diakonessen Hospital Utrecht, Utrecht, The Netherlands \\ ${ }^{2}$ Department of Surgery, University Medical Center Utrecht, Utrecht, The Netherlands \\ ${ }^{3}$ Department of Nuclear Medicine, Diakonessen Hospital Utrecht, Utrecht, The Netherlands \\ ${ }^{4}$ Department of Internal Medicine, Diakonessen Hospital Utrecht, Utrecht, The Netherlands \\ Email: batwigt@gmail.com
}

Received October 4, 2012; revised November 2, 2012; accepted November 12, 2012

\begin{abstract}
Background: The objective of this study was to assess the additional yield of US and CT following a "negative" initial MIBI-scintigraphy (MIBI) in patients with primary hyperparathyroidism. Methods: Prospective data were collected regarding 100 consecutive patients, preferentially undergoing a minimally invasive parathyroidectomy (MIP). MIBI was the initial imaging study for localizing a solitary adenoma, followed by US and CT (US/CT) in "MIBI-negative"-patients. Results: Surgery led to normocalcemia in 98 patients (98\%) after one operation. Overall 97 patients had solitary parathyroid disease while three patients had multiglandular disease. The sensitivity of imaging increased from $74 \%$ for MIBI alone to $92 \%$ following subsequent US/CT in "MIBI-negative"-patients. The positive predictive value of a "positive" MIBI was $96 \%$ and $76 \%$ of a positive US/CT following negative MIBI. The proportion of patients who underwent successful MIP increased from 60 to $72 \%$. Conclusions: MIBI and the combination of US and CT are complementary imaging studies. Additional localization studies after a negative sestamibi scan enhances the number of patients with primary hyperparathyroidism profiting from a minimally invasive approach.
\end{abstract}

Keywords: Imaging-Primary Hyperparathyroidism-Sestamibi Scan-Ultrasound-CT

\section{Introduction}

Primary hyperparathyroidism (PHPT) affects $0.3 \%$ of the general population and the incidence is 21.6 cases per 100,000 person-years $[1,2]$. The incidence rises with age and women are affected twice as much as men. In the last two decades minimally invasive parathyroidectomy (MIP) has gradually replaced conventional neck exploration (CNE) as the surgical procedure of choice in patients with sporadic primary hyperparathyroidism (pHPT). MIP reduces the extent of surgical dissection, operative time, hospital stay and perioperative morbidity, [3-7] while cure rates are comparable to the results of CNE [8]. Preoperative parathyroid adenoma localization and intra-operative PTH-assessment (IOPTH) both contributed to this success $[9,10]$.

Correct preoperative imaging of a solitary adenoma is a prerequisite for a focused surgical approach. Preoperative imaging strategies varies. Tc-99-sestamibi scintigraphy (MIBI) is most commonly used and frequently advocated as the initial investigation [11]. Its sensitivity is reported as high as $71 \%-93 \%$ [12-17]. While MIBI identifies a hyperfunctional parathyroid gland (PG), ultrasonography (US) and CT-scanning (CT) of the neck detect an enlarged PG. At present single-photon emission computed tomography (SPECT) and the fusion of SPECT and CT images (SPECT/CT) is gaining importance, combining qualities that aim to detect a physiological abnormality and determine its exact anatomical localization [18].

In order to maximize the potential number of candidates for minimally invasive parathyroidectomy we routinely use MIBI as a first investigational step, followed by CT and US (CT/US) when MIBI is "negative", in patients with sporadic pHPT. In a prospective cohort study, the additional yield of CT/US following a "negative" MIBI was evaluated.

\section{Methods}

From January 2000 until September 2010 data were collected prospectively of all patients operated for pHPT in the Diakonessenhuis Hospital Utrecht. Patients with familial hyperparathyroidism (MEN-syndromes), patients previously operated for pHPT and lithium induced hyperparathyroidism were excluded. In all patients the diagnosis was established biochemically by: an increased serum calcium level $(>10.20 \mathrm{mg} / \mathrm{dL})$ combined with an 
increased $(>70 \mathrm{pg} / \mathrm{mL})$ or a not suppressed plasma PTH level, or an increased renal calcium excretion combined with an elevated PTH level.

Planar parathyroid scintigraphy using ${ }^{99 \mathrm{~m}} \mathrm{Tc}$-sestamibi (MIBI) was routinely done as a first investigational step for localization of a solitary adenoma. When MIBI scanning revealed no adenoma both US (13 $\mathrm{MHz}$ lineair transducer; Acuson Antares, Siemens) and CT (16 slice; Somatom, Siemens) using a slice thickness of $3 \mathrm{~mm}$ interval of the neck were done (CT/US). All patients were operated under general anaesthesia by the same surgeon. When at least one investigational procedure suggested a solitary adenoma a minimal invasive operation was started with a small incision that could be converted into a Kochers incision when necessary. A MIP was started as a 2-cm-long transverse incision at the medial border of the sternocleidomastoid muscle and continued as a "lateral approach" [19]. Concomitant thyroid pathology, suspicion of parathyroid malignancy and large size of a parathyroid adenoma were reasons for a unilateral exploration through a $4 \mathrm{~cm}$ Kochers incision, without exploration of the contralateral glands.

After removal of a preoperatively identified abnormal gland, the operation was ended, without further exploration of the neck and identifying the other parathyroid glands. When the intraoperative findings were not consistent with the preoperative imaging, MIP was converted to a CNE. IOPTH sampling was not available. Intraoperative frozen section analysis was used to confirm the parathyroid origin of excised tissue specimens. Gland weight was determined to reveal a possible relation between weight and MIBI sensitivity. Patients were cured when serum calcium and PTH levels normalized postoperatively and remained normal at least six months after definitive surgery.

\section{Statistical Analysis}

Since all imaging studies aimed to localise a solitary adenoma, a positive imaging result was defined as the visualisation of a single parathyroid abnormality. Patients were classified as having single gland disease when they were cured after removal of one abnormal PG. A contingency $(2 \times 2)$ table was made relating the operative findings to the preoperative predicted localization. Sensitivity of imaging studies aiming to detect solitary adenoma was defined as the proportion of patients with solitary gland disease (solitary adenoma and carcinoma) in whom a solitary adenoma was identified and correctly localized by preoperative imaging (no effort was made to distinguish between superior or inferior glands).

Positive predictive value (PPV) was defined as the proportion of all patients with a positive imaging study in whom the result had correctly identified and localized single gland disease.
The overall success rate of the preoperative imaging work-up was defined as the proportion of all patients in whom a solitary adenoma was correctly identified, i.e. in accordance with the outcome of the operation.

The additional yield of US/CT following a "negative" MIBI was addressed by evaluating its effect on the overall sensitivity of the preoperative imaging work-up, as well as the positive predictive value of US/CT as compared to MIBI alone and the effect on the proportion of patients who underwent successful minimal invasive surgery.

\section{Results}

One hundred consecutive patients underwent parathyroid surgery for non-familial pHPT. There where 23 men and 77 women, with a median age of 60 years (range 25 - 85). Patient characteristics are listed in Table 1.

Preoperative MIBI showed unilateral uptake consistent with a solitary adenoma in 75 of 100 patients (75\%) (Figure 1). In one patient scintigraphy suggested MGD. In the other 24 patients additional US/CT suggested the presence of a solitary adenoma in 21 patients. Overall, scintigraphy, followed by CT/US identified 96 patients with a presumed solitary adenoma.

MIP was the planned operative approach in 91 out of the 96 patients with visualized solitary gland disease. In 17 patients (18\%) MIP was converted to a CNE due to insufficient exposure in eleven patients and preoperative imaging not being consistent with the intraoperative findings in six. The success rate of the first operation was 98 percent. As a result of the first operative procedure one enlarged PG was removed in 97 patients, two or more enlarged PG were found in two patients, and no

Table 1. Characteristics of 100 patients operated for primary hyperparathyroidism.

\begin{tabular}{cc}
\hline & Number of patients $(\mathrm{n}=100)$ \\
\hline Male:female & $23: 77$ \\
Median age (range) & $60(25-85)$ \\
Frequency of symptoms & 55 \\
Fatigue & 22 \\
Renalstones & 41 \\
Osteoporosis & 21 \\
Abdominal complaints & 13 \\
Psychic changes & \\
& \\
& \\
Mean preoperative (range) & \\
Calcium (mg/dL) & $11.92(9.16-22.20)$ \\
PTH (pg/mL) & $219(26-1810)$ \\
\hline
\end{tabular}




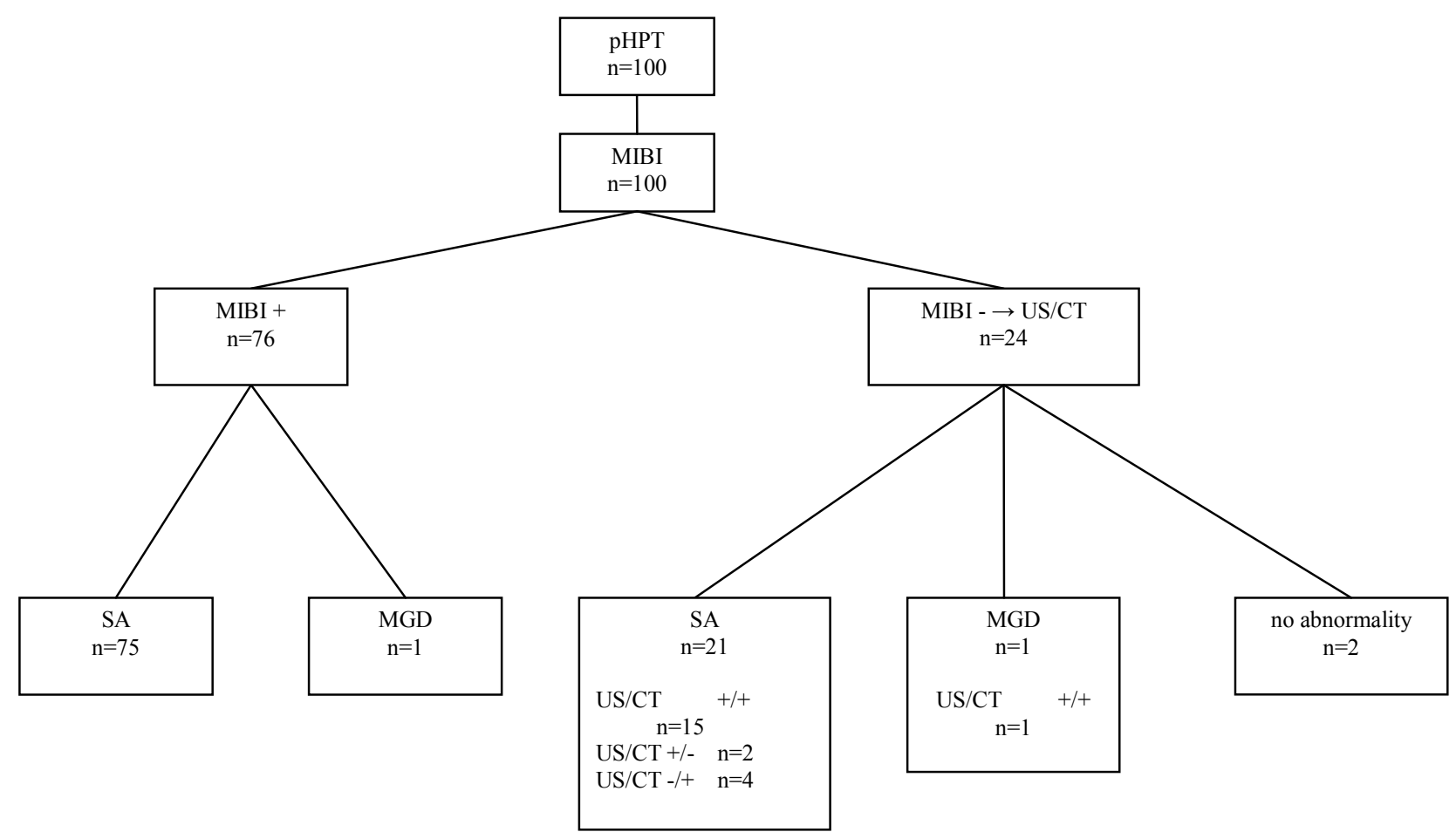

Figure 1. pHPT; primary hyperparathyroidism, SA; solitary adenoma, MGD; multiglandular disease, MIBI; MIBI scintigraphy, US; ultrasound, CT; CT scan.

adenoma was found in one patient. Hypercalcemia persisted in two patients $(2 \%)$. The patient in whom no adenoma had been found was subsequently operated elsewhere where a single adenoma was retrieved (consistent with the preoperative MIBI). The other patient underwent a second operation which revealed a second (larger) adenoma.

The overall incidence of solitary adenoma was $96 \%$, multiglandular disease 3\% and carcinoma $1 \%$. Postoperative complications included one permanent recurrent laryngeal nerve damage following a MIP (1\%), two transient recurrent laryngeal nerve palsy's $(2 \%)$ and 2 postoperative haematomas (2\%) both requiring surgical re-exploration.

The operative findings correlated with a preoperative MIBI suggesting a solitary adenoma in 72 of the 75 patients (PPV $=96 \%)$. In 16 of 21 patients who had a $\mathrm{CT} / \mathrm{US}$ visualizing an assumed solitary adenoma, a single parathyroid abnormality was retrieved accordingly (PPV $=76 \%$ ). When US and CT unequivocally identified one parathyroid abnormality $(\mathrm{n}=16)$, the operative findings were consistent with these investigations in ten patients. In eleven out of 100 patients imaging did not correlate with the intraoperative findings (Table 2).

The sensitivity of preoperative imaging to correctly identify a solitary adenoma increased from $74 \%$ after MIBI alone to $92 \%$ following additional CT and US. At the same time, the overall PPV decreased from 96 to 90
Table 2. Summary of patients in whom imaging did not correlate with operative findings $(n=11)$.

\begin{tabular}{|c|c|c|c|}
\hline $\mathrm{n}$ & Imaging result & Imaging studies & Intraoperative findings \\
\hline 4 & SA & $\begin{array}{c}\text { MIBI + } \\
n=1 \\
\text { MIBI-, US/CT + } \\
n=3\end{array}$ & $\begin{array}{l}\text { All patients had a SA, bu } \\
\text { not on the predicted loca- } \\
\text { tion } n=4\end{array}$ \\
\hline 3 & SA & $\begin{array}{c}\text { MIBI + } \\
n=2 \\
\text { MIBI-, US/CT + } \\
n=1\end{array}$ & $\begin{array}{l}\text { All patients had MGD } \\
\qquad \mathrm{n}=3\end{array}$ \\
\hline 2 & MGD & $\begin{array}{c}\text { MIBI: MGD } \\
\mathrm{n}=1 \\
\text { MIBI-, US/CT: } \\
\text { MGD } \mathrm{n}=1\end{array}$ & $\begin{array}{l}\text { Both patients had a SA } \\
\qquad \mathrm{n}=2\end{array}$ \\
\hline 2 & No abnormality & $\begin{array}{c}\text { MIBI-, US/CT - } \\
n=2\end{array}$ & $\begin{array}{l}\text { Both patients had a SA } \\
\qquad \mathrm{n}=2\end{array}$ \\
\hline
\end{tabular}

percent respectively. The workup success rate of our strategy was $89 \%$ and the rate of successful MIP increased from 60 to $72 \%$.

\section{Discussion}

In the present study the additional yield of ultrasound and CT after a negative scintigraphy was evaluated. Following a "negative" MIBI scintigraphy in one quarter of the patients, subsequent US and CT suggested a solitary adenoma in the majority of them. The proportion of patients with correctly identified solitary adenomas increased from $75 \%$ to $92 \%$, and the proportion of patients 
who underwent successful minimally invasive parathyroidectomy from $60 \%$ to $72 \%$.

The strength of the present study is its prospective design and the adherence to an algorithm using MIBI-scintigraphy, neck ultrasound and CT stepwise to identify solitary adenomas in patients with pHPT. All three imaging techniques are readily available techniques.

The main weakness is the limited number of patients, making firm conclusions difficult. Then again, it does reflect the ability to achieve a good surgical success rate in a modest volume setting even in MIBI "negative" patients. The observed surgical success rate in a modest volume institution contradicts the conclusions from others to treat MIBI "negative" patients only in high volume institutions [20]. Furthermore we did not investigate the contemporary use of SPECT or SPECT/CT, however MIBI is a readily available technique and was used in our hospital in the past decade. Lastly, the proportion of patients with multiglandular disease was low in the present study, which is in line with our recent multi-institutional study observing a significantly lower incidence of multiglandular disease than previously reported [21].

Preoperative parathyroid adenoma localization is a prerequisite for a focused surgical approach in patients with pHPT and many imaging techniques and strategies are available and used for that purpose. MIBI-scans, ultrasonography, MRI, CT, SPECT are all used in the work-up of patients with $\mathrm{pHPT}$ with reported sensitivities ranging between $20 \%$ and $96 \%$.

High cure rates in patients with primary hyperparathyroidectomy and two positive imaging studies are described $[22,23]$. Many authors have reported on MIBIscintigraphy in combination with neck ultrasonography [24-28]. Ultrasonography has the advantage of being a readily available, cheap, preoperative localization study without the use of radiation, but it is strongly operator depended and the resulting image is difficult to interpret by the operating surgeon. CT has the benefit of providing the exact localization (even in an ectopic localization) of the enlarged gland thereby providing a roadmap for the operating surgeon, but at the expense of radiation to a vulnerable area (thyroid gland) and higher costs. The yield of the combination of ultrasound and MIBI ranges between $48 \%$ and $94 \%$. Then again, in many of these studies it is unclear what the contribution of the separate techniques is, and surgical success rate is commonly confused with imaging identification rate (i.e. sensitivity). In addition, different surgical approaches (bilateral, unilateral or focused) and poor description of correct identification (side or quadrant) makes comparison of results difficult.

Applying the stepwise approach described in the present study, using US and CT when MIBI is negative has two advantages. On the one hand, the increased overall sensitivity of $92 \%$ in the present study is accompanied by an increased number of patients selected for, and successfully operated by a minimally invasive procedure. On the other hand, by using US and CT only when the scintigraphy was negative, unnecessary investigations are not done in the majority of patients with a positive MIBI. The positive predictive value of $96 \%$ when a MIBI-scan is showing unilateral uptake justifies this approach. The contemporary use of SPECT or SPECT/CT may further increase this percentage in the near future, but it is accompanied by an increase in costs and patient radiation doses [18].

The increasing number of false positive imaging studies appears to be a disadvantage of the algorithm. The chance of a false positive investigation increases when the MIBI is negative, and even more when the subsequent US and CT show equivocal results. Awareness of this decreasing reliability of a positive imaging study is important and should be communicated with the patient. Nevertheless, our data demonstrate that there is little if no harm of an opportunistic minimally invasive exploration; it selects two-third of patients who had a "negative" MIBI who can profit from a MIP. One may argue that the conversion rate in this group was high, but these patients would have had an upfront CNE anyway if the CT/US had not been done in addition.

Pushing the limits by the stepwise use of readily available imaging techniques increases the identification rate of solitary adenomas in patients with pHPT and selects more patients for minimally invasive surgery.

\section{REFERENCES}

[1] R. A. Wermers, S. Khosla, E. J. Atkinson, S. J. Achenbach, A. L. Oberg, C. S. Grant and L. J. Melton III, "Incidence of Primary Hyperparathyroidism in Rochester, Minnesota, 1993-2001: An Update on the Changing Epidemiology of the Disease," Journal of Bone and Miner Research, Vol. 21, No. 1, 2006, pp. 171-177. doi:10.1359/JBMR.050910

[2] L. J. Melton III, "The Epidemiology of Primary Hyperparathyroidism in North America," Journal of Bone and Miner Research, Vol. 17, Suppl. 2, 2002, pp. N12-N17.

[3] A. Bergenfelz, P. Lindblom, S. Tibblin and J. Westerdahl, "Unilateral versus Bilateral Neck Exploration for Primary Hyperparathyroidism: A Prospective Randomized Controlled Trial," Annals of Surgery, Vol. 236, No. 5, 2002, pp. 543-551. doi:10.1097/00000658-200211000-00001

[4] R. E. Goldstein, L. Blevins, D. Delbeke and W. H. Martin, "Effect of Minimally Invasive Radioguided Parathyroidectomy on Efficacy, Length of Stay, and Costs in the Management of Primary Hyperparathyroidism," Annals of Surgery, Vol. 231, No. 5, 2000, pp. 732-742. doi:10.1097/00000658-200005000-00014

[5] R. Udelsman, "Six Hundred Fifty-Six Consecutive Explorations for Primary Hyperparathyroidism," Annals of 
Surgery, Vol. 235, No. 5, 2002, pp. 665-670. doi:10.1097/00000658-200205000-00008

[6] K. Lorenz, P. Nguyen-Thanh and H. Dralle, "Unilateral Open and Minimally Invasive Procedures for Primary Hyperparathyroidism: A Review of Selective Approaches," Langenbeck's Archives of Surgery, Vol. 385, No. 2, 2000, pp. 106-117. doi:10.1007/s004230050252

[7] R. A. Low and A. D. Katz, "Parathyroidectomy via Bilateral Cervical Exploration: A Retrospective Review of 866 Cases," Head \& Neck, Vol. 20, No. 7, 1998, pp. 583-587. doi:10.1002/(SICI)1097-0347(199810)20:7<583::AID-H ED1>3.0.CO;2-X

[8] C. S. Grant, G. Thompson, D. Farley and J. van Heerden, "Primary Hyperparathyroidism Surgical Management since the Introduction of Minimally Invasive Parathyroidectomy: Mayo Clinic Experience," Archives of Surgery, Vol. 140, No. 5, 2005, pp. 472-478.

doi:10.1001/archsurg. 140.5.472

[9] G. L. Irvin III, A. S. Molinari, C. Figueroa and D. M. Carneiro, "Improved Success Rate in Reoperative Parathyroidectomy with Intraoperative PTH Assay," Annals of Surgery, Vol. 229, No. 6, 1999, pp. 874-878. doi:10.1097/00000658-199906000-00015

[10] W. R. Sackett, B. Barraclough, T. S. Reeve and L. W. Delbridge, "Worldwide Trends in the Surgical Treatment of Primary Hyperparathyroidism in the Era of Minimally Invasive Parathyroidectomy," Archives of Surgery, Vol. 137, 2002, pp. 1055-1059.

doi:10.1001/archsurg.137.9.1055

[11] C. Y. Lo, B. H. Lang, W. F. Chan, A. W. Kung and K. S. Lam, "A Prospective Evaluation of Preoperative Localization by Technetium-99m Sestamibi Scintigraphy and Ultrasonography in Primary Hyperparathyroidism," American Journal of Surgery, Vol. 193, No. 2, 2007, pp. 155-159. doi:10.1016/j.amjsurg.2006.04.020

[12] A. Caixas, L. Berna, A. Hernandez, F. J. Tebar, P. Madariaga, O. Vegazo, A. L. Bittini, B. Moreno, E. Faure, D. Abos, J. Piera, J. M. Rodriguez, J. Farrerons and M. Puig-Domingo, "Efficacy of Preoperative Diagnostic Imaging Localization of Technetium 99m-Sestamibi Scintigraphy in Hyperparathyroidism," Surgery, Vol. 121, No. 5, 1997, pp. 535-541. doi:10.1016/S0039-6060(97)90108-2

[13] A. C. Civelek, E. Ozalp, P. Donovan and R. Udelsman, "Prospective Evaluation of Delayed Technetium-99m Sestamibi SPECT Scintigraphy for Preoperative Localization of Primary Hyperparathyroidism," Surgery, Vol. 131, No. 2, 2002, pp. 149-157. doi:10.1067/msy.2002.119817

[14] M. Gotthardt, B. Lohmann, T. M. Behr, A. Bauhofer, C. Franzius, M. L. Schipper, M. Wagner, H. Hoffken, H. Sitter, M. Rothmund, K. Joseph and C. Nies, "Clinical Value of Parathyroid Scintigraphy with Technetium-99m Methoxyisobutylisonitrile: Discrepancies in Clinical Data and a Systematic Metaanalysis of the Literature," World Journal of Surgery, Vol. 28, No. 1, 2004, pp. 100-107. doi:10.1007/s00268-003-6991-y

[15] R. S. Haber, C. K. Kim and W. B. Inabnet, "Ultrasonography for Preoperative Localization of Enlarged Parathyroid Glands in Primary Hyperparathyroidism: Com- parison with (99m)Technetium Sestamibi Scintigraphy," Clinical Endocrinology, Vol. 57, No. 2, 2002, pp. 241249.

[16] A. Malhotra, C. E. Silver, V. Deshpande and L. M. Freeman, "Preoperative Parathyroid Localization with Sestamibi," American Journal of Surgery, Vol. 172, No. 6, 1996, pp. 637-640. doi:10.1016/S0002-9610(96)00289-9

[17] M. J. O’Doherty, A. G. Kettle, P. Wells, R. E. Collins and A. J. Coakley, "Parathyroid Imaging with Technetium99m-Sestamibi: Preoperative Localization and Tissue Uptake Studies," Journal of Nuclear Medicine, Vol. 33, No. 3, 1992, pp. 313-318.

[18] M. L. Taubman, M. Goldfarb and J. I. Lew, "Role of SPECT and SPECT/CT in the Surgical Treatment of Primary Hyperparathyroidism," International Journal of Molecular Imaging, Vol. 2011, 2011, Article ID: 141593. doi: $10.1155 / 2011 / 141593$

[19] P. C. Smit, I. Rinkes, A. van Dalen and T. J. van Vroonhoven, "Direct, Minimally Invasive Adenomectomy for Primary Hyperparathyroidism: An Alternative to Conventional Neck Exploration?" Annals of Surgery, Vol. 231, No. 4, 2000, pp. 559-565. doi:10.1097/00000658-200004000-00016

[20] D. M. Elaraj, R. S. Sippel, S. Lindsay, I. Sansano, Q. Y. Duh, O. H. Clark and E. Kebebew, "Are Additional Localization Studies and Referral Indicated for Patients with Primary Hyperparathyroidism Who Have Negative Sestamibi Scan Results?" Archives of Surgery, Vol. 145, No. 6, 2010, pp. 578-581. doi:10.1001/archsurg.2010.108

[21] B. Twigt, A. Vollebregt and T. van Dalen, "Shifting Incidence of Solitary Adenomas in the Era of Minimally Invasive Parathyroidectomy. A Multi-Institutional Study," Annals of Surgical Oncology, Vol. 18, No. 4, 2010, pp. 1041-1046. doi:10.1245/s10434-010-1394-4

[22] M. Barczynski, A. Konturek, S. Cichon, A. HubalewskaDydejczyk, F. Golkowski and B. Huszno, "Intraoperative Parathyroid Hormone Assay Improves Outcomes of Minimally Invasive Parathyroidectomy Mainly in Patients with a Presumed Solitary Parathyroid Adenoma and Missing Concordance of Preoperative Imaging," Clinical Endocrinology, Vol. 66, No. 6, 2007, pp. 878-885. doi:10.1111/j.1365-2265.2007.02827.x

[23] A. A. Gawande, J. M. Monchik, T. A. Abbruzzese, J. D. Iannuccilli, S. I. Ibrahim and F. D. Moore Jr., "Reassessment of Parathyroid Hormone Monitoring during Parathyroidectomy for Primary Hyperparathyroidism after 2 Preoperative Localization Studies," Archives of Surgery, Vol. 141, No. 4, 2006, pp. 381-384. doi:10.1001/archsurg.141.4.381

[24] C. Arici, W. K. Cheah, P. H. Ituarte, E. Morita, T. C. Lynch, A. E. Siperstein, Q. Y. Duh and O. H. Clark, "Can Localization Studies Be Used to Direct Focused Parathyroid Operations?" Surgery, Vol. 129, No. 6, 2001, pp. 720-729. doi:10.1067/msy.2001.114556

[25] R. S. Haber, C. K. Kim and W. B. Inabnet, "Ultrasonography for Preoperative Localization of Enlarged Parathyroid Glands in Primary Hyperparathyroidism: Comparison with (99m)Technetium Sestamibi Scintigraphy," Clinical Endocrinology, Vol. 57, No. 2, 2002, pp. 241- 
249. doi:10.1046/j.1365-2265.2002.01583.x

[26] G. P. Purcell, F. M. Dirbas, R. B. Jeffrey, M. J. Lane, T. Desser, I. R. McDougall and R. J. Weigel, "Parathyroid Localization with High-Resolution Ultrasound and Technetium Tc 99m Sestamibi," Archives of Surgery, Vol. 134, 1999, pp. 824-828. doi:10.1001/archsurg.134.8.824

[27] M. O. Saint, A. Cogliandolo, R. R. Pidoto and A. Pozzo, "Prospective Evaluation of Ultrasonography Plus MIBI Scintigraphy in Selecting Patients with Primary Hyper- parathyroidism for Unilateral Neck Exploration under Local anaesthesia," American Journal of Surgery, Vol. 187, No. 3, 2004, pp. 388-393. doi:10.1016/j.amjsurg.2003.12.013

[28] L. S. Freudenberg, A. Frilling, S. Y. Sheu and R. Gorges, "Optimizing Preoperative Imaging in Primary Hyperparathyroidism," Langenbeck's Archives of Surgery, Vol. 391, No. 6, 2006, pp. 551-556. doi:10.1007/s00423-006-0076-y 